\title{
Impact of Plant-Based Antimicrobial Washes on Sensory Properties of Organic Leafy Greens
}

\author{
Kamini Joshi' ${ }^{1}$ Patricia Sparks' ${ }^{2}$, Mendel Friedman ${ }^{3}$, Sadhana Ravishankar ${ }^{{ }^{*}}$ \\ ${ }^{1}$ School of Animal and Comparative Biomedical Sciences, University of Arizona, Tucson, AZ, USA \\ ${ }^{2}$ Department of Nutritional Sciences, University of Arizona, Tucson, AZ, USA \\ ${ }^{3}$ USDA-ARS-WRRC, Albany, CA, USA \\ Email: "sadhravi@email.arizona.edu
}

Received 19 July 2016; accepted 28 August 2016; published 31 August 2016

Copyright (C) 2016 by authors and Scientific Research Publishing Inc.

This work is licensed under the Creative Commons Attribution International License (CC BY).

http://creativecommons.org/licenses/by/4.0/

(c) () D Den Access

\section{Abstract}

The objective was to study the sensory attributes of organic leafy greens treated with plant antimicrobials and identify treatments most accepted by panelists. Organic leafy greens were washed with antimicrobials and stored at $4^{\circ} \mathrm{C}$ for $24 \mathrm{~h}$ prior to serving panelists. Antimicrobials evaluated include: $0.1 \%$ clove bud, lemongrass, oregano, or cinnamon essential oils; $0.1 \%$ carvacrol or citral; $3 \%$ grapeseed, apple, or $10 \% / 7 \%$ olive extract; combination of essential oils with extracts; $3 \%$ hydrogen peroxide; and untreated control. A randomized block design with an affective test was used and 60 panelists were asked to evaluate samples for preference liking based on a 9-point hedonic scale and for sensory attributes based on a 5-point hedonic scale. Changes in texture and color of leafy greens were measured using a Texture analyzer and a Chroma Meter, respectively. On the basis of preference liking, overall acceptability of spinach and lettuce treated with $0.1 \%$ cinnamon oil was ranked the highest (7.5 \pm 1.4 and $7.1 \pm 1.7$, moderately liked), respectively. For texture analysis, washing iceberg lettuce with $0.1 \%$ oregano oil $+10 \%$ olive extract and spinach with $0.1 \%$ lemongrass oil $+1 \%$ apple extract yielded the highest firmness values of $F=783.1 \pm$ 53.8 Newtons and $939.30 \pm 35.2$ Newtons, respectively. Based on the International Commission on Illumination CIE LAB color schemes, treatment with $0.1 \%$ oregano oil $+10 \%$ olive extract had the greatest impact on color of iceberg lettuce with the lowest $L$ value* $(44.5 \pm 6.2)$ indicating the darkest color. These results will help identify plant antimicrobials that have the least impact on sensory properties of organic leafy greens and are preferred by consumers.

\section{Keywords}

Plant Antimicrobials, Organic Leafy Greens, Sensory Analysis, Texture Analysis, Color Measurements

\footnotetext{
"Corresponding author.
}

How to cite this paper: Joshi, K., Sparks, P., Friedman, M. and Ravishankar, S. (2016) Impact of Plant-Based Antimicrobial Washes on Sensory Properties of Organic Leafy Greens. Food and Nutrition Sciences, 7, 906-919. 


\section{Introduction}

Organic foods have recently gained popularity among consumers because they are perceived to be more environmentally friendly [1], nutritious, and health promoting than conventionally grown foods [2] [3]. As of 2012, the sales of organic food products in the United States have reached a market of $\$ 28.4$ billion, which is $4 \%$ of the total food sales [4]. The top selling category among organic food products is fruits and vegetables [4]. As the consumption of organic food has increased, there is also a concern about increased risk of foodborne illnesses. Previous studies have shown that organically grown produce has higher microbial load than conventionally grown produce [5]. The organic food industry is limited in its sanitation options, particularly in the usage of chemical-based sanitizers; therefore, alternative organic sanitization options such as plant antimicrobials in the wash water are being evaluated.

Essential oils (EOs) are gaining popularity as potential organic sanitizers or preservatives because they have broad-spectrum antimicrobial activity and are considered "Generally Recognized as Safe" (GRAS) [6]. There is an increased interest in natural antimicrobials compared to chemical preservatives due to many health concerns. Previous in vitro studies have shown that EOs have antibacterial activity against Listeria monocytogenes, Salmonella Typhimurium, Escherichia coli O157:H7, Shigella dysenteriae, Bacillus cereus and Staphylococcus aureus at concentrations of $0.2-10 \mu \mathrm{l} / \mathrm{ml}$ [7]. If these EOs are to be used as natural preservatives or disinfectants, their organoleptic properties on treated foods should be considered, because they can alter the taste or flavor of the food [8]. A sensory appeal is one of the most important factors that will determine food choices by consumers [9]. A study showed that EOs not only reduced the pathogen load during storage in meat samples, but also improved the organoleptic properties of meat [10].

It has been shown that treatment with $1 \mathrm{mM}$ carvacrol or cinnamic acid showed no significant adverse effects on the organoleptic attributes of kiwi fruits and honeydew melons [11]. Citrus oil components such as limonene, and 1-octanol had no effect on the aroma quality of some foods [12]. There was no significant difference between vegetables washed with chlorine and EOs based on color, texture, and water activity of the samples as well as the gas composition in the package [13]. No significant difference was found between lettuces washed with $250 \mathrm{ppm}$ oregano oil versus that washed with chlorinated water, as evaluated by a sensory panel [13]. In vegetable soup, the acceptable concentration for rosemary, thyme, carvacrol, or $p$-cymene was $20 \mu \mathrm{L} / \mathrm{L}$, whereas for lemon oil it was $200 \mu \mathrm{L} / \mathrm{L}$ [14].

The characteristics that impact the quality of fruits and vegetables are described by four attributes: 1) color and appearance; 2) flavor (taste and aroma); 3) texture; and 4) nutritional value [15]. In order to avoid the undesirable organoleptic effects of EOs on food, careful selection of these compounds is necessary. In this study, we therefore tested various essential oils, their active components, plant extracts, both alone and in combinations to determine both preference liking by consumers and effects on the sensory properties of organic leafy greens. Due to the increased popularity of natural treatments, especially in the organic industry, this study focused on investigating concerns related to sensory acceptability of plant-based antimicrobials on organic leafy greens.

\section{Materials and Methods}

\subsection{Plant Antimicrobials Used}

EOs used in this study included oregano oil, lemongrass oil, clove bud oil, and cinnamon oil; the active components used included carvacrol, and citral. The plant extracts evaluated were apple, grapeseed, and olive extract. Oregano oil (100\% pure Origanum vulgare), and clove bud oil (100\% pure Eugenia caryophyllata) were obtained from Lhasa Karnak Herb Company (Berkeley, CA, USA). Cinnamon oil (100\% pure Cinnamomum cassia) and lemongrass oil (100\% pure Cymbopogon flexuosus) were obtained from Now Foods (Bloomingdale, IL, USA). Natural citral (96\%), and carvacrol (99\%) were obtained from Sigma Aldrich® (St. Louis, MO, USA). Olive extract (Olea europaea) HIDROX ${ }^{\circledR} 10 \mathrm{x}$ in liquid concentrate was obtained from CreAgri, Inc. (Hayward, CA, USA). Grapeseed extract was obtained from Swanson Health products (Fargo, ND, USA). Apple skin extract was obtained from Apple Poly LLC. (Morrill, NE). $\mathrm{H}_{2} \mathrm{O}_{2}(3 \%)$ was purchased from the local retail stores.

All treatment solutions were made in tap water in their respective chosen concentrations. The following plant antimicrobials were evaluated for preference liking and specific sensory attributes on organic baby spinach and iceberg lettuce samples: $0.1 \%$ oregano oil, $0.1 \%$ lemongrass oil, $0.1 \%$ clove bud oil, $0.1 \%$ cinnamon oil, $0.1 \%$ carvacrol, $0.1 \%$ citral, $3 \%$ apple extract, $3 \%$ grapeseed extract, $7 \%$ olive extract, $10 \%$ olive extract (the concentration for olive extract is higher because this is a liquid extract as opposed to others in the powder form), and 
various combinations of EOs with plant extracts. $\mathrm{H}_{2} \mathrm{O}_{2}(3 \%)$ was used as a control.

\subsection{Treatments of Organic Leafy Greens}

Organic iceberg lettuce and baby spinach were purchased from a local retail store the same day of use. Organic leafy greens were thoroughly washed with tap water and air-dried. Lettuce and spinach samples were washed in water $(300 \mathrm{ml})$ containing the respective concentrations of plant antimicrobials for 2 min with gentle agitation done manually. After treatment, the leafy green samples were stored at $4^{\circ} \mathrm{C}$ for $20-24 \mathrm{~h}$. A control (washed in tap water without any antimicrobials) was included during each trial.

\subsection{Sensory Analysis}

Sensory analysis was performed using 60 untrained panelists during each trial. A total of 6 trials were conducted ( 3 for lettuce and 3 for spinach). A randomized block design with affective test was conducted to generate data for preference liking [16]. Each panelist was provided with $1 \mathrm{~g}$ of sample per treatment and was asked to drink water to clean his/her palette and wait at least 2 min prior to evaluating the next sample. Panelists were asked to evaluate each sample for preference liking based on the 9-point hedonic scale where, $9=$ like extremely, $8=$ like very much, $7=$ like moderately, $6=$ like slightly, $5=$ neither like nor dislike, $4=$ dislike slightly, $3=$ dislike moderately, 2 = dislike very much, and $1=$ dislike extremely. Sensory parameters for preference liking were: aroma, color, freshness, mouthfeel, flavor, and overall acceptability. Panelists were also asked to quantify each sample for sensory attributes including pungency, browning, bitterness, off-odor, and sourness based on a 5point hedonic scale where 1 was rated the lowest and 5 the highest $(1=$ not pungent, $2=$ slightly pungent, $3=$ moderately pungent, $4=$ very pungent and $5=$ extremely pungent).

The sensory study was conducted at the sensory lab in the Department of Nutritional Sciences, University of Arizona. Constant yellow lights were used in the sensory booths allowing panelists to evaluate visual differences among samples. Panelists also answered questions about demographics such as age, gender, ethnicity, frequency of consumption of organic/conventionally grown leafy greens, and the types of leafy greens they would like to purchase (iceberg lettuce, romaine lettuce, spinach, or mixed greens).

\subsection{Texture Analysis}

The texture of each antimicrobial treated leafy green was measured using an Instron or Texture Analyzer (Texture Lab Pro, provided by Food Technology Corporation, Sterling, VA, USA). The leafy greens were treated as described earlier with the appropriate concentration of each antimicrobial or their combinations and stored at $4^{\circ} \mathrm{C}$ for 20 - $24 \mathrm{~h}$ prior to measurements. A $1000 \mathrm{~N}$ load cell was attached to a Kramer shear cell with an 8-blade probe attached to the instrument with the speed set to $250 \mathrm{~mm} / \mathrm{min}$ [17]. A sample $(15 \mathrm{~g})$ of treated leafy greens was placed in the Kramer cell chamber to determine the crispiness/firmness value. Three repeats were conducted and an average of the highest peak force (measured in Newtons $(\mathrm{N})$ ) from three trials was indicated as the crispiness value of the sample.

\subsection{Color Measurements}

The color of the treated leafy greens was measured using a Minolta Chroma Meter (Model CR-400, Minolta, Inc., Tokyo, Japan). The color was measured using the International Commission on Illumination-CIE L*, a*, and $b^{*}$ coordinates. The $\mathrm{L}^{*}$ value is a measurement of lightness from dark $\left(\mathrm{L}^{*}=0\right)$ to absolute light $\left(\mathrm{L}^{*}=100\right)$; $\mathrm{a}^{*}$ axis ranges from green $(-)$ to red $(+)$; and $\mathrm{b}^{*}$ axis ranges from blue $(-)$ to yellow $(+)[18]$. The instrument was calibrated using Minolta standard white reflector plate. Four repeats of the experiment were conducted and three different readings were taken during each repeat (total readings 12) for each sample.

\subsection{Statistical Analysis}

For sensory analysis, each experiment was divided into three trials with new sets of 60 panelists at each trial. A randomized block design was used for sensory analysis. An average was calculated based on 60 responses for preference liking and sensory characteristics for each treatment. Data were analyzed using One-way Analysis of variance (ANOVA) Tukey's pairwise test at a level of significance of $p \leq 0.05$ using Minitab 17 (State College, PA, USA). A linear regression was used to determine the correlation between overall acceptability and other 
sensory parameters based on preference liking. Statistical analysis on texture and color measurements was also done using ANOVA, with statistical level of significance considered at $\mathrm{p} \leq 0.05$.

\section{Results and Discussions}

Numerous studies have shown antimicrobial properties of plant-based compounds (19-22); however, studies concerning their effect on the sensory properties of leafy greens have been limited. One study showed that the addition of oregano essential oil preserved the intensity rating of positive attributes in extra virgin olive oil during storage [23]. To our knowledge, the impact of plant antimicrobial washes on the sensory attributes of organic leafy greens has not been extensively studied. Therefore, in this study, we identified plant antimicrobial washes for organic leafy greens that had the highest preference liking among consumers, and described how the color and firmness attributes of leafy greens are impacted by these compounds.

The concentration of each antimicrobial for washing organic leafy greens was chosen based on previous work indicating its antimicrobial properties. A total of 15 different treatments were evaluated for lettuce and 16 different treatments for spinach samples. Sensory analyses on lettuce samples were conducted first; therefore, treatments that were considerably disliked by panelists were not further tested on spinach samples. In addition, preliminary work was conducted using higher concentrations of plant antimicrobials for sensory analysis; however, due to majority of consumers' dislike preferences, lower concentrations were chosen for this study.

Affective test is a sensory test commonly used to identify samples with the highest preference liking when comparing multiple samples using untrained panelists [24]. Hence, we chose this type of sensory test for our study. Additionally, we asked the panelists to quantify the level of pungency, browning, bitterness, off-odor, and sourness noticed in an antimicrobial treated leafy green sample. To our knowledge, extensive sensory studies have not been conducted on organic leafy greens washed with plant antimicrobials; this study can therefore be used as a baseline to identify sensory characteristics of these samples.

\subsection{Demographics Information of the Panelists}

The criteria for the panelists to participate in this study included the following: 1) each panelist must be at least 18 years or older; 2) must be a non-smoker; and 3) be able to differentiate between colors. Results for all demographic information are shown in Figure 1. A total of 360 responses from panelists were obtained in this study (180 responses were obtained for a total of 15 lettuce samples and another 180 for a total of 16 spinach samples). Of the 360 participants, $75 \%$ were female, and $25 \%$ were male. The age of the panelists ranged from 18 to 78 years, with majority of them $(65 \%)$ in the age range $18-25$ years. On the basis of ethnicity, the panelists included 47\% White, 24\% Hispanic or Latino, 22\% Asian, 3\% African American, and 4\% others. The highest preference concerning the types of leafy greens was for spinach (35\%), followed by mixed greens (34\%), romaine $(22 \%)$, and iceberg lettuce (9\%). Of the participants, $56 \%$ indicated that they consume conventionally grown leafy greens at least twice a week and 24\% indicated that they consume organic leafy greens at least twice a week. Another similar study showed that overall, there were no significant differences between the consumer liking and consumer perceived sensory qualities of organically and conventionally grown vegetables including tomatoes, cucumbers, and onions [25].

\subsection{Sensory Analysis of Organic Iceberg Lettuce Treated with Plant Antimicrobials}

Among all plant antimicrobial treatments, iceberg lettuce washed with $0.1 \%$ cinnamon oil had the highest preference liking by panelists, ranging from 6 to 7 on the hedonic scale (liked slightly/moderately). Cinnamon oil may be preferred by panelists as there is a sweet taste associated with it. Another possible reason could be the familiarity of cinnamon among consumers. Majority of the population in this study were 18 - 25 years old white females, which may also describe the higher preference liking for cinnamon oil compared to others. Sweetness taste has higher hedonic appeal especially among children and young people [26]. A study conducted using 1005 participants from North America showed that females preferred comfort foods such as chocolate and ice cream compared to males [27]. For aroma, color, freshness, mouthfeel, flavor, and overall acceptability, there were no significant differences $(\mathrm{p} \leq 0.05)$ between control, $3 \% \mathrm{H}_{2} \mathrm{O}_{2}$, and $0.1 \%$ cinnamon oil treatments for iceberg lettuce samples (Table 1). Additionally, other treatments that were slightly or moderately preferred by panelists include: $0.1 \%$ clove bud oil, $0.1 \%$ citral, and $0.1 \%$ oregano oil. Treatment of organic iceberg lettuce with $0.1 \%$ 


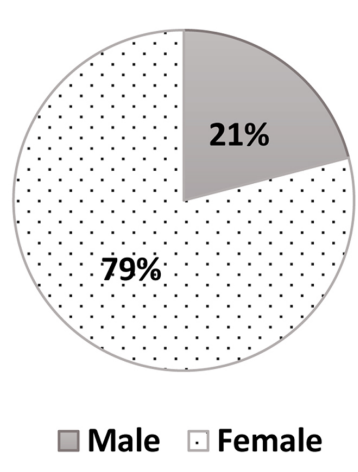

(a)

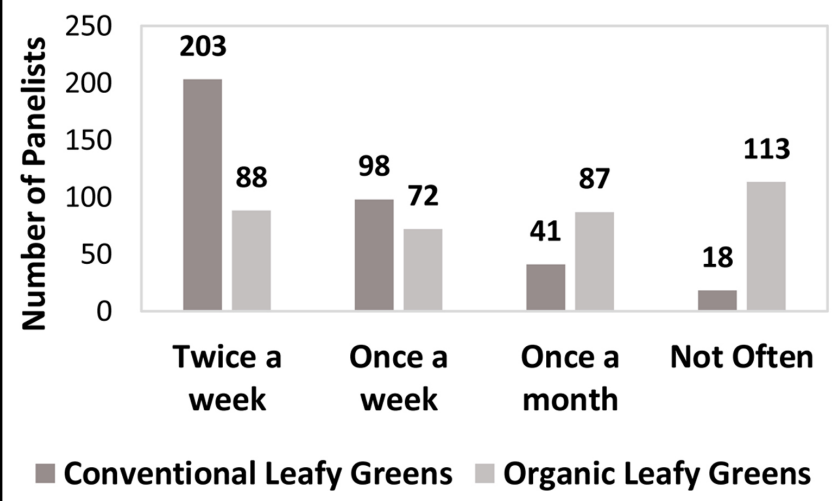

(c)

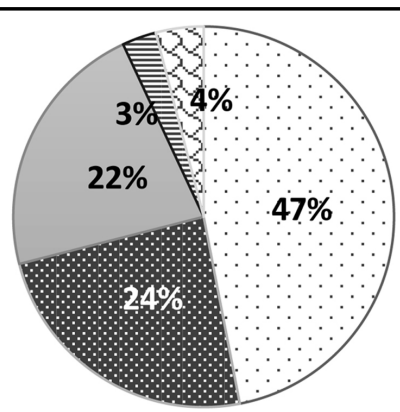

$\square$ White Hispanic $\square$ Asian 目African American $\supset$ Other

(b)

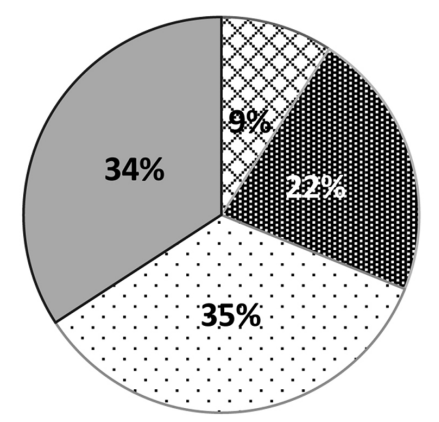

$\square$ Iceberg 여 Romaine $\square$ Spinach $\square$ Mixed Greens

(d)

Figure 1. Demographic information on the panelists who participated in the sensory study for both organic iceberg lettuce and baby spinach washed with plant antimicrobials. Data depict information obtained from a total of 360 responses of which 180 were for iceberg lettuce samples and 180 for baby spinach samples. (a) Gender ratio; (b) Ethnicity ratio; (c) Pattern for consumption of leafy greens; (d) Preference for type of leafy greens.

cinnamon oil had the least impact on pungency, browning, bitterness, off-odor, and sourness of lettuce (Table 2). Other studies have indicated similar results where vegetables (iceberg lettuce, beet, and arugula) sanitized with Origanum vulgare and Rosmarinus officinalis essential oils alone and in combination were "liked slightly" and "neither liked nor disliked" based on the 5-point hedonic scale [28].

Combination treatments and treatments with plant extracts had the highest influence on preference liking by consumers based on color, as these treatments imparted higher browning characteristics to iceberg lettuce. Customers evaluate visual appearance and color first followed by taste, aroma, and texture [15]. EOs or active components used (individually) for washing iceberg lettuce did not have a significant $(\mathrm{p} \leq 0.05)$ impact on browning, since there were no significant differences between the control, $3 \% \mathrm{H}_{2} \mathrm{O}_{2}, 0.1 \%$ cinnamon oil, $0.1 \%$ citral, and $0.1 \%$ oregano oil, all of which had a rating level of 1 (not brown at all).

Washing iceberg lettuce with tap water (control), $3 \% \mathrm{H}_{2} \mathrm{O}_{2}, 3 \%$ grapeseed extract, or $3 \%$ apple extract did not have a significant impact $(\mathrm{p} \leq 0.05)$ on the pungency level, as all samples were rated at level 1 , not pungent at all. The majority of the treatments evaluated did not increase the bitterness or sourness level of iceberg lettuce in comparison to the control or $3 \% \mathrm{H}_{2} \mathrm{O}_{2}$. Again, samples that were not significantly $(\mathrm{p} \leq 0.05)$ different based on off-odor in comparison to the control or $3 \% \mathrm{H}_{2} \mathrm{O}_{2}$ included those that were treated with $0.1 \%$ cinnamon oil.

\subsection{Sensory Analysis of Organic Baby Spinach Treated with Plant Antimicrobials}

For spinach samples, the treatment with the highest preference liking based on aroma was $0.1 \%$ lemongrass, and based on all other parameters (color, freshness, mouthfeel, flavor, and overall acceptability) $0.1 \%$ cinnamon oil had the highest preference liking (Table 3). The treatments that had an adverse impact on the color parameters 
Table 1. Preference liking for organic iceberg lettuce samples washed with essential oils, plant extracts, or combination of both. The data depicted is an average of 60 responses for each treatment \pm standard deviation. Different letters signify statistical differences between various treatments $(\mathrm{p} \leq 0.05)$ for each sensory parameter.

\begin{tabular}{|c|c|c|c|c|c|c|}
\hline Treatments & Aroma & Color & Freshness & Mouthfeel & Flavor & $\begin{array}{c}\text { Overall } \\
\text { acceptability }\end{array}$ \\
\hline Control & $6.55 \pm 1.56^{\mathrm{A}}$ & $7.40 \pm 1.65^{\mathrm{A}}$ & $7.70 \pm 1.60^{\mathrm{ABC}}$ & $7.73 \pm 1.38^{\mathrm{A}}$ & $7.15 \pm 1.98^{\mathrm{A}}$ & $7.53 \pm 1.48^{\mathrm{A}}$ \\
\hline $3 \% \mathrm{H}_{2} \mathrm{O}_{2}$ & $6.35 \pm 1.89^{\mathrm{A}}$ & $7.82 \pm 1.69^{\mathrm{A}}$ & $7.97 \pm 1.34^{\mathrm{AB}}$ & $7.78 \pm 1.57^{\mathrm{A}}$ & $7.30 \pm 1.67^{\mathrm{A}}$ & $7.48 \pm 1.37^{\mathrm{A}}$ \\
\hline $0.1 \%$ Cinnamon oil & $6.83 \pm 1.69^{\mathrm{A}}$ & $7.49 \pm 1.60^{\mathrm{A}}$ & $8.25 \pm 0.94^{\mathrm{A}}$ & $7.71 \pm 1.45^{\mathrm{A}}$ & $6.59 \pm 1.90^{\mathrm{AB}}$ & $7.12 \pm 1.67^{\mathrm{AB}}$ \\
\hline $0.1 \%$ Clove bud oil & $5.73 \pm 2.11^{\mathrm{ABC}}$ & $6.02 \pm 1.96^{\mathrm{B}}$ & $6.75 \pm 1.75^{\mathrm{BCD}}$ & $7.02 \pm 1.70^{\mathrm{AB}}$ & $5.53 \pm 2.46^{\mathrm{BCDE}}$ & $5.97 \pm 1.96^{\mathrm{BC}}$ \\
\hline $0.1 \%$ Lemongrass oil & $5.85 \pm 2.70^{\mathrm{ABC}}$ & $6.00 \pm 2.21^{\mathrm{B}}$ & $5.95 \pm 2.26^{\mathrm{DEF}}$ & $6.13 \pm 1.90^{\mathrm{BCD}}$ & $4.80 \pm 2.56^{\mathrm{CDEF}}$ & $5.27 \pm 2.24^{\mathrm{CDE}}$ \\
\hline $0.1 \%$ Oregano oil & $5.00 \pm 2.46^{\mathrm{BCD}}$ & $7.43 \pm 1.77^{\mathrm{A}}$ & $7.05 \pm 1.79^{\mathrm{ABCD}}$ & $6.95 \pm 1.96^{\mathrm{AB}}$ & $5.08 \pm 2.53^{\mathrm{CDEF}}$ & $5.65 \pm 2.29^{C}$ \\
\hline 0.1\% Carvacrol & $4.88 \pm 2.15^{\mathrm{BCD}}$ & $6.76 \pm 2.03^{\mathrm{AB}}$ & $6.53 \pm 2.27^{\mathrm{CDE}}$ & $5.75 \pm 2.63^{\mathrm{BCDE}}$ & $3.65 \pm 2.18^{\mathrm{FG}}$ & $4.20 \pm 2.32^{\mathrm{DEF}}$ \\
\hline 0.1\% Citral & $6.07 \pm 2.62^{\mathrm{AB}}$ & $7.56 \pm 1.76^{\mathrm{A}}$ & $7.22 \pm 2.09^{\mathrm{ABC}}$ & $6.78 \pm 2.35^{\mathrm{AB}}$ & $5.66 \pm 2.58^{\mathrm{BCD}}$ & $5.70 \pm 2.44^{\mathrm{C}}$ \\
\hline 3\% Apple extract & $5.80 \pm 1.80^{\mathrm{ABC}}$ & $2.80 \pm 1.86^{\mathrm{CDE}}$ & $4.25 \pm 2.62^{\mathrm{GH}}$ & $5.77 \pm 2.21^{\mathrm{BCDE}}$ & $5.53 \pm 2.26^{\mathrm{BCDE}}$ & $4.78 \pm 2.11^{\mathrm{CDE}}$ \\
\hline 3\% Grapeseed extract & $5.90 \pm 1.63^{\mathrm{ABC}}$ & $3.23 \pm 1.49^{\mathrm{CD}}$ & $5.40 \pm 2.23^{\mathrm{EFG}}$ & $6.42 \pm 1.84^{\mathrm{BC}}$ & $6.03 \pm 2.21^{\mathrm{ABC}}$ & $5.28 \pm 1.68^{\mathrm{CD}}$ \\
\hline $7 \%$ Olive extract & $5.00 \pm 2.02^{\mathrm{BCD}}$ & $2.98 \pm 1.93^{\mathrm{CDE}}$ & $4.07 \pm 2.12^{\mathrm{H}}$ & $5.00 \pm 2.41^{\mathrm{DE}}$ & $4.16 \pm 2.46^{\mathrm{EF}}$ & $3.52 \pm 2.06^{\mathrm{F}}$ \\
\hline $10 \%$ Olive extract & $5.68 \pm 1.92^{\mathrm{ABC}}$ & $3.45 \pm 2.02^{\mathrm{C}}$ & $3.78 \pm 2.15^{\mathrm{H}}$ & $4.80 \pm 2.20^{\mathrm{E}}$ & $4.30 \pm 2.49^{\mathrm{DEF}}$ & $4.25 \pm 2.10^{\mathrm{DEF}}$ \\
\hline $\begin{array}{c}0.1 \% \text { Clove bud oil }+3 \% \\
\text { Apple extract }\end{array}$ & $4.17 \pm 2.87^{\mathrm{DE}}$ & $2.13 \pm 1.62^{\mathrm{DE}}$ & $4.09 \pm 2.32^{\mathrm{H}}$ & $5.27 \pm 2.20^{\mathrm{CDE}}$ & $3.83 \pm 2.31^{\mathrm{F}}$ & $3.52 \pm 1.99^{\mathrm{F}}$ \\
\hline $\begin{array}{l}0.1 \% \text { Oregano oil }+1 \% \\
\text { Grapeseed extract }\end{array}$ & $4.73 \pm 2.12^{\mathrm{CD}}$ & $3.64 \pm 2.13^{\mathrm{C}}$ & $4.71 \pm 2.23^{\mathrm{FGH}}$ & $5.21 \pm 2.32^{\mathrm{CDE}}$ & $3.98 \pm 2.48^{\mathrm{F}}$ & $4.02 \pm 2.31^{\mathrm{EF}}$ \\
\hline $\begin{array}{l}0.1 \% \text { Oregano oil }+10 \% \\
\text { Olive extract }\end{array}$ & $3.35 \pm 2.27^{\mathrm{E}}$ & $1.93 \pm 1.44^{\mathrm{E}}$ & $2.47 \pm 2.04^{\mathrm{I}}$ & $2.98 \pm 2.01^{\mathrm{F}}$ & $2.27 \pm 2.00^{\mathrm{G}}$ & $2.25 \pm 1.73^{\mathrm{G}}$ \\
\hline
\end{tabular}

Table 2. Impact of plant antimicrobials on the sensory characteristics of organic iceberg lettuce evaluated by panelists. Data represent an average of 60 responses for each sample \pm standard deviation. Average values which do not share the same letter, are significantly different $(\mathrm{p} \leq 0.05)$.

\begin{tabular}{|c|c|c|c|c|c|}
\hline Treatments & Pungency & Browning & Bitterness & Off-Odor & Sourness \\
\hline Control & $1.11 \pm 0.33^{\mathrm{G}}$ & $1.18 \pm 0.50^{\mathrm{EFG}}$ & $1.31 \pm 0.72^{\mathrm{GH}}$ & $1.08 \pm 0.33^{\mathrm{F}}$ & $1.03 \pm 0.18^{\mathrm{G}}$ \\
\hline $3 \% \mathrm{H}_{2} \mathrm{O}_{2}$ & $1.33 \pm 0.68^{\mathrm{FG}}$ & $1.14 \pm 0.51^{\mathrm{FG}}$ & $1.48 \pm 0.77^{\mathrm{FGH}}$ & $1.15 \pm 0.52^{\mathrm{F}}$ & $1.20 \pm 0.51^{\mathrm{EFG}}$ \\
\hline 0.1\% Cinnamon oil & $1.83 \pm 0.94^{\mathrm{EF}}$ & $1.05 \pm 0.22^{\mathrm{G}}$ & $1.28 \pm 0.59^{\mathrm{H}}$ & $1.72 \pm 0.90^{\mathrm{EF}}$ & $1.13 \pm 0.39^{\mathrm{FG}}$ \\
\hline $0.1 \%$ Clove bud oil & $2.93 \pm 1.10^{\mathrm{BCD}}$ & $1.62 \pm 0.80^{\mathrm{DE}}$ & $1.90 \pm 1.02^{\mathrm{DEFGH}}$ & $2.78 \pm 1.19^{\mathrm{BCD}}$ & $1.34 \pm 0.74^{\mathrm{DEFG}}$ \\
\hline $0.1 \%$ Lemongrass oil & $3.57 \pm 1.27^{\mathrm{AB}}$ & $1.88 \pm 0.96^{\mathrm{D}}$ & $1.98 \pm 1.07^{\mathrm{CDEFG}}$ & $3.40 \pm 1.22^{\mathrm{AB}}$ & $1.92 \pm 1.03^{\mathrm{BCD}}$ \\
\hline $0.1 \%$ Oregano oil & $2.93 \pm 1.35^{\mathrm{BCD}}$ & $1.30 \pm 0.62^{\mathrm{EFG}}$ & $2.17 \pm 1.38^{\mathrm{BCDE}}$ & $2.60 \pm 1.37^{\mathrm{CD}}$ & $1.51 \pm 0.89^{\mathrm{CDEFG}}$ \\
\hline $0.1 \%$ Carvacrol & $2.73 \pm 1.07^{\mathrm{D}}$ & $1.55 \pm 0.81^{\mathrm{DEF}}$ & $2.53 \pm 1.37^{\mathrm{BCD}}$ & $2.90 \pm 1.07^{\mathrm{ABC}}$ & $1.75 \pm 1.02^{\mathrm{CDE}}$ \\
\hline $0.1 \%$ Citral & $2.73 \pm 1.25^{\mathrm{D}}$ & $1.27 \pm 0.58^{\mathrm{EFG}}$ & $1.62 \pm 0.94^{\mathrm{EFGH}}$ & $2.85 \pm 1.16^{\mathrm{BCD}}$ & $1.59 \pm 0.93^{\mathrm{CDEFG}}$ \\
\hline 3\% Apple extract & $1.25 \pm 0.60^{\mathrm{FG}}$ & $3.65 \pm 0.92^{\mathrm{B}}$ & $1.71 \pm 1.00^{\mathrm{EFGH}}$ & $1.30 \pm 0.53^{\mathrm{F}}$ & $1.37 \pm 0.66^{\mathrm{DEFG}}$ \\
\hline $3 \%$ Grapeseed extract & $1.28 \pm 0.55^{\mathrm{FG}}$ & $3.22 \pm 0.96^{\mathrm{BC}}$ & $1.62 \pm 0.87^{\mathrm{EFGH}}$ & $1.32 \pm 0.57^{\mathrm{F}}$ & $1.35 \pm 0.73^{\mathrm{DEFG}}$ \\
\hline $7 \%$ Olive extract & $2.38 \pm 1.17^{\mathrm{DE}}$ & $3.65 \pm 0.95^{\mathrm{B}}$ & $2.46 \pm 1.21^{\mathrm{BCD}}$ & $2.43 \pm 1.14^{\mathrm{CD}}$ & $1.97 \pm 1.16^{\mathrm{BC}}$ \\
\hline $10 \%$ Olive extract & $2.48 \pm 1.03^{\mathrm{D}}$ & $3.17 \pm 0.83^{\mathrm{C}}$ & $2.70 \pm 1.27^{\mathrm{B}}$ & $2.23 \pm 1.10^{\mathrm{DE}}$ & $2.40 \pm 1.17^{\mathrm{AB}}$ \\
\hline $\begin{array}{l}0.1 \% \text { Clove bud oil }+3 \% \\
\text { Apple extract }\end{array}$ & $3.43 \pm 1.20^{\mathrm{ABC}}$ & $4.27 \pm 0.84^{\mathrm{A}}$ & $2.63 \pm 1.24^{\mathrm{BC}}$ & $3.37 \pm 1.22^{\mathrm{AB}}$ & $1.60 \pm 1.03^{\mathrm{CDEFG}}$ \\
\hline $\begin{array}{l}0.1 \% \text { Oregano oil }+1 \% \\
\text { Grapeseed extract }\end{array}$ & $2.88 \pm 1.19^{\mathrm{CD}}$ & $3.23 \pm 0.85^{\mathrm{BC}}$ & $2.14 \pm 1.18^{\mathrm{BCDEF}}$ & $2.98 \pm 1.19^{\mathrm{ABC}}$ & $1.67 \pm 1.02^{\mathrm{CDEF}}$ \\
\hline $\begin{array}{l}0.1 \% \text { Oregano oil }+10 \% \\
\text { Olive extract }\end{array}$ & $3.62 \pm 1.14^{\mathrm{A}}$ & $4.37 \pm 0.76^{\mathrm{A}}$ & $3.45 \pm 1.28^{\mathrm{A}}$ & $3.52 \pm 1.21^{\mathrm{A}}$ & $2.87 \pm 1.49^{\mathrm{A}}$ \\
\hline
\end{tabular}


based on preference liking were: $3 \%$ grapeseed extract, $0.1 \%$ clove bud oil $+3 \%$ apple extract, $0.1 \%$ oregano oil $+1 \%$ grapeseed extract, $0.1 \%$ carvacrol, and $0.1 \%$ oregano oil $+7 \%$ olive extract. Treatments that gave slightly brown $(2.0-2.3)$ color to baby spinach included: $3 \%$ grapeseed, $0.1 \%$ clove bud oil $+3 \%$ apple extract, $0.1 \%$ oregano oil $+1 \%$ grapeseed extract, and $0.1 \%$ oregano oil $+7 \%$ olive extract. All other treatments were rated not brown at all $(1.1-1.7)$. The following treatments did not have significantly different $(\mathrm{p} \leq 0.05)$ pungency or off-odor levels in comparison to the control and $3 \% \mathrm{H}_{2} \mathrm{O}_{2}: 0.1 \%$ clove bud oil, $3 \%$ apple extract, $3 \%$ grapeseed extract, and a combination of $0.1 \%$ clove bud oil $+3 \%$ apple extract; they all had a rating of 1 (not pungent/ off-odor at all). Treatments that were significantly different $(\mathrm{p} \geq 0.05)$ based on bitterness included: $0.1 \%$ oregano oil $+1 \%$ grapeseed extract, $0.1 \%$ oregano oil, $7 \%$ olive extract, $0.1 \%$ carvacrol, and $0.1 \%$ oregano oil + $7 \%$ olive extract. Treatments that significantly $(\mathrm{p} \geq 0.05)$ impacted the sourness value of spinach included: $0.1 \%$ citral, $0.1 \%$ lemongrass oil $+1 \%$ apple extract, $0.1 \%$ oregano oil, $7 \%$ olive extract, $0.1 \%$ carvacrol, and combination of $0.1 \%$ oregano $+7 \%$ olive extract which had ratings of $1.9 \pm 1.1-2.1 \pm 1.2$ (slightly sour).

The least preferred treatment for spinach samples was a combination of $7 \%$ olive extract with $0.1 \%$ oregano oil based on aroma, flavor and overall acceptability. For color preference, spinach treated with $3 \%$ grapeseed extract had the lowest rating $(6.0 \pm 2.4$, like slightly), and for freshness, $0.1 \%$ clove bud oil $+3 \%$ apple extract had the lowest rating $(5.8 \pm 2.5$, neither like nor dislike); however, the difference was not significant $(\mathrm{p} \leq 0.05)$ between these treatments and $7 \%$ olive extract $+0.1 \%$ oregano oil. The combination of olive extract with oregano oil was the least preferred based on overall acceptability $(4.4 \pm 2.5$, slightly dislike), with this treatment being ranked the highest for pungency (3.2 \pm 1.2$)$, browning (2.0 \pm 0.9$)$, and bitterness (3.0 \pm 1.3$)$ (Table 4). There is a negative correlation between rating of preference liking and sensory characteristics including pungency, browning, bitterness, off-odor, and sourness (data not shown).

Table 3. Preference liking for organic baby spinach samples washed with essential oils, plant extracts, or combination of both. The data depicted is an average of 60 responses for each treatment \pm standard deviation. Different letters signify statistical differences between various treatments $(\mathrm{p} \leq 0.05)$ for each sensory parameter.

\begin{tabular}{|c|c|c|c|c|c|c|}
\hline Treatments & Aroma & Color & Freshness & Mouthfeel & Flavor & $\begin{array}{c}\text { Overall } \\
\text { Acceptability }\end{array}$ \\
\hline Control & $6.93 \pm 2.02^{\mathrm{ABC}}$ & $8.00 \pm 1.35^{\mathrm{AB}}$ & $7.50 \pm 1.77^{\mathrm{AB}}$ & $6.93 \pm 1.78^{\mathrm{ABC}}$ & $6.86 \pm 1.76^{\mathrm{A}}$ & $7.14 \pm 1.46^{\mathrm{AB}}$ \\
\hline $3 \% \mathrm{H}_{2} \mathrm{O}_{2}$ & $6.83 \pm 1.92^{\mathrm{ABCD}}$ & $7.78 \pm 1.70^{\mathrm{AB}}$ & $7.32 \pm 2.06^{\mathrm{ABC}}$ & $6.97 \pm 1.85^{\mathrm{ABC}}$ & $6.29 \pm 2.42^{\mathrm{AB}}$ & $6.65 \pm 2.05^{\mathrm{ABC}}$ \\
\hline $0.1 \%$ Cinnamon oil & $7.10 \pm 1.77^{\mathrm{AB}}$ & $8.18 \pm 1.10^{\mathrm{A}}$ & $7.92 \pm 1.51^{\mathrm{A}}$ & $7.47 \pm 1.33^{\mathrm{A}}$ & $6.57 \pm 2.09^{\mathrm{AB}}$ & $7.48 \pm 1.37^{\mathrm{A}}$ \\
\hline $0.1 \%$ Clove bud oil & $7.10 \pm 1.79^{\mathrm{AB}}$ & $8.17 \pm 1.14^{\mathrm{A}}$ & $7.80 \pm 1.35^{\mathrm{A}}$ & $7.27 \pm 1.75^{\mathrm{AB}}$ & $6.58 \pm 2.14^{\mathrm{AB}}$ & $6.95 \pm 1.89^{\mathrm{ABC}}$ \\
\hline $0.1 \%$ Lemongrass oil & $7.30 \pm 1.80^{\mathrm{A}}$ & $8.03 \pm 1.05^{\mathrm{AB}}$ & $7.78 \pm 1.39^{\mathrm{A}}$ & $7.19 \pm 1.54^{\mathrm{ABC}}$ & $5.51 \pm 2.18^{\mathrm{ABCD}}$ & $6.24 \pm 1.83^{\mathrm{ABCD}}$ \\
\hline $0.1 \%$ Oregano oil & $5.63 \pm 2.69^{\mathrm{CDEF}}$ & $7.20 \pm 1.74^{\mathrm{ABCD}}$ & $6.70 \pm 1.78^{\mathrm{ABCDEF}}$ & $5.93 \pm 2.18^{\mathrm{CDE}}$ & $4.30 \pm 2.52^{\mathrm{DE}}$ & $4.93 \pm 2.37^{\mathrm{DEF}}$ \\
\hline 0.1\% Carvacrol & $5.48 \pm 2.56^{\mathrm{EF}}$ & $7.00 \pm 2.11^{\mathrm{BCDE}}$ & $6.05 \pm 2.28^{\mathrm{DEF}}$ & $5.50 \pm 2.50^{\mathrm{DE}}$ & $4.50 \pm 2.68^{\mathrm{CDE}}$ & $4.58 \pm 2.44^{\mathrm{EF}}$ \\
\hline $0.1 \%$ Citral & $6.72 \pm 2.36^{\mathrm{ABCDE}}$ & $7.42 \pm 1.96^{\mathrm{ABC}}$ & $6.85 \pm 2.02^{\mathrm{ABCDEF}}$ & $6.02 \pm 2.20^{\mathrm{BCDE}}$ & $5.58 \pm 2.14^{\mathrm{ABCD}}$ & $5.88 \pm 2.12^{\mathrm{BCDE}}$ \\
\hline 3\% Apple extract & $6.77 \pm 1.89^{\mathrm{ABCDE}}$ & $7.30 \pm 1.94^{\mathrm{ABC}}$ & $7.00 \pm 1.90^{\mathrm{ABCDE}}$ & $6.88 \pm 1.80^{\mathrm{ABC}}$ & $6.35 \pm 1.98^{\mathrm{AB}}$ & $6.50 \pm 2.19^{\mathrm{ABC}}$ \\
\hline 3\% Grapeseed extract & $6.60 \pm 1.88^{\mathrm{ABCDE}}$ & $5.95 \pm 2.38^{\mathrm{E}}$ & $6.05 \pm 2.20^{\mathrm{DEF}}$ & $6.29 \pm 2.04^{\mathrm{ABCDE}}$ & $5.63 \pm 2.36^{\mathrm{ABCD}}$ & $5.80 \pm 2.15^{\mathrm{BCDE}}$ \\
\hline $7 \%$ Olive extract & $5.93 \pm 2.32^{\mathrm{BCDEF}}$ & $7.12 \pm 1.72^{\mathrm{ABCD}}$ & $6.41 \pm 2.08^{\mathrm{BCDEF}}$ & $5.32 \pm 2.41^{\mathrm{E}}$ & $4.19 \pm 2.48^{\mathrm{DE}}$ & $4.59 \pm 2.27^{\mathrm{EF}}$ \\
\hline $\begin{array}{l}0.1 \% \text { Cinnamon oil }+1 \% \\
\text { Grapeseed extract }\end{array}$ & $6.88 \pm 2.00^{\mathrm{ABC}}$ & $7.22 \pm 1.70^{\mathrm{ABCD}}$ & $6.95 \pm 1.83^{\mathrm{ABCDEF}}$ & $6.77 \pm 1.78^{\mathrm{ABCD}}$ & $5.28 \pm 2.34^{\mathrm{BCDE}}$ & $5.82 \pm 2.01^{\mathrm{BCDE}}$ \\
\hline $\begin{array}{c}0.1 \% \text { Clove bud oil }+3 \% \\
\text { Apple extract }\end{array}$ & $6.55 \pm 1.87^{\mathrm{ABCDE}}$ & $5.93 \pm 2.27^{\mathrm{E}}$ & $5.77 \pm 2.48^{\mathrm{F}}$ & $6.20 \pm 2.21^{\mathrm{BCDE}}$ & $5.62 \pm 2.51^{\mathrm{ABCD}}$ & $5.68 \pm 2.40^{\mathrm{CDEF}}$ \\
\hline $\begin{array}{c}0.1 \% \text { Lemongrass }+1 \% \\
\text { Apple extract }\end{array}$ & $6.65 \pm 2.22^{\mathrm{ABCDE}}$ & $7.57 \pm 1.58^{\mathrm{ABC}}$ & $7.08 \pm 1.92^{\mathrm{ABCD}}$ & $6.83 \pm 2.00^{\mathrm{ABC}}$ & $5.90 \pm 2.50^{\mathrm{ABC}}$ & $6.17 \pm 2.42^{\mathrm{ABCD}}$ \\
\hline $\begin{array}{c}0.1 \% \text { Oregano }+1 \% \\
\text { Grapeseed extract }\end{array}$ & $5.53 \pm 2.13^{\mathrm{DEF}}$ & $6.13 \pm 2.18^{\mathrm{DE}}$ & $5.78 \pm 2.28^{\mathrm{EF}}$ & $5.40 \pm 2.21^{\mathrm{E}}$ & $4.63 \pm 2.23^{\mathrm{CDE}}$ & $5.03 \pm 2.12^{\mathrm{DEF}}$ \\
\hline $\begin{array}{c}0.1 \% \text { Oregano oil }+7 \% \\
\text { Olive Extract }\end{array}$ & $5.10 \pm 2.31^{\mathrm{F}}$ & $6.62 \pm 2.00^{\mathrm{CDE}}$ & $6.27 \pm 2.19^{\mathrm{CDEF}}$ & $5.93 \pm 2.22^{\mathrm{CDE}}$ & $3.92 \pm 2.44^{\mathrm{E}}$ & $4.36 \pm 2.48^{\mathrm{F}}$ \\
\hline
\end{tabular}


Table 4. Impact of plant antimicrobials on the sensory characteristics of organic baby spinach evaluated by panelists. Data represent an average of 60 responses for each sample \pm standard deviation. Average values which do not share the same letter, are significantly different $(\mathrm{p} \leq 0.05)$.

\begin{tabular}{|c|c|c|c|c|c|}
\hline Treatments & Pungency & Browning & Bitterness & Off-Odor & Sourness \\
\hline Control & $1.21 \pm 0.52^{\mathrm{G}}$ & $1.13 \pm 0.39^{\mathrm{DE}}$ & $1.43 \pm 0.67^{\mathrm{E}}$ & $1.23 \pm 0.59^{\mathrm{F}}$ & $1.27 \pm 0.58^{\mathrm{C}}$ \\
\hline $3 \% \mathrm{H}_{2} \mathrm{O}_{2}$ & $1.18 \pm 0.39^{\mathrm{G}}$ & $1.25 \pm 0.51^{\mathrm{CDE}}$ & $1.72 \pm 0.83^{\mathrm{DE}}$ & $1.23 \pm 0.56^{\mathrm{F}}$ & $1.48 \pm 0.77^{\mathrm{BC}}$ \\
\hline $0.1 \%$ Cinnamon oil & $1.90 \pm 1.12^{\mathrm{EF}}$ & $1.13 \pm 0.50^{\mathrm{DE}}$ & $1.51 \pm 0.88^{\mathrm{DE}}$ & $1.75 \pm 1.04^{\mathrm{CDEF}}$ & $1.27 \pm 0.61^{\mathrm{C}}$ \\
\hline $0.1 \%$ Clove bud oil & $1.40 \pm 0.69^{\mathrm{FG}}$ & $1.08 \pm 0.28^{\mathrm{E}}$ & $1.60 \pm 0.83^{\mathrm{DE}}$ & $1.52 \pm 0.85^{\mathrm{DEF}}$ & $1.52 \pm 0.81^{\mathrm{ABC}}$ \\
\hline $0.1 \%$ Lemongrass oil & $2.85 \pm 1.19^{\mathrm{ABC}}$ & $1.23 \pm 0.50 \mathrm{C}^{\mathrm{DE}}$ & $1.95 \pm 0.86^{\mathrm{CDE}}$ & $2.50 \pm 1.16^{\mathrm{AB}}$ & $1.82 \pm 1.07^{\mathrm{ABC}}$ \\
\hline $0.1 \%$ Oregano oil & $3.25 \pm 1.19^{\mathrm{A}}$ & $1.33 \pm 0.60^{\mathrm{CDE}}$ & $2.60 \pm 1.28^{\mathrm{AB}}$ & $2.98 \pm 1.29^{\mathrm{A}}$ & $1.98 \pm 1.19^{\mathrm{AB}}$ \\
\hline $0.1 \%$ Carvacrol & $2.97 \pm 1.16^{\mathrm{AB}}$ & $1.58 \pm 1.02^{\mathrm{BCD}}$ & $2.51 \pm 1.12^{\mathrm{ABC}}$ & $2.98 \pm 1.18^{\mathrm{A}}$ & $2.05 \pm 1.17^{\mathrm{AB}}$ \\
\hline $0.1 \%$ Citral & $2.67 \pm 1.32^{\mathrm{ABCD}}$ & $1.38 \pm 0.76^{\mathrm{CDE}}$ & $1.80 \pm 0.98^{\mathrm{DE}}$ & $2.48 \pm 1.36^{\mathrm{AB}}$ & $1.93 \pm 1.09^{\mathrm{AB}}$ \\
\hline 3\% Apple extract & $1.38 \pm 0.85^{\mathrm{FG}}$ & $1.60 \pm 0.72^{\mathrm{BCD}}$ & $1.58 \pm 0.83^{\mathrm{DE}}$ & $1.37 \pm 0.81^{\mathrm{DEF}}$ & $1.72 \pm 1.01^{\mathrm{ABC}}$ \\
\hline 3\% Grapeseed extract & $1.28 \pm 0.61^{\mathrm{FG}}$ & $2.18 \pm 0.89^{\mathrm{A}}$ & $1.58 \pm 0.83^{\mathrm{DE}}$ & $1.33 \pm 0.60^{\mathrm{EF}}$ & $1.52 \pm 0.81^{\mathrm{ABC}}$ \\
\hline $7 \%$ Olive extract & $2.05 \pm 1.14^{\mathrm{DE}}$ & $1.68 \pm 0.83^{\mathrm{BC}}$ & $3.03 \pm 1.26^{\mathrm{A}}$ & $1.92 \pm 1.00^{\mathrm{BCDE}}$ & $2.12 \pm 1.21^{\mathrm{A}}$ \\
\hline $0.1 \%$ Cinnamon oil + $1 \%$ Grapeseed extract & $2.33 \pm 1.15^{\mathrm{CDE}}$ & $1.67 \pm 0.77^{\mathrm{BC}}$ & $1.98 \pm 1.05^{\mathrm{BCDE}}$ & $2.50 \pm 1.24^{\mathrm{AB}}$ & $1.58 \pm 0.87^{\mathrm{ABC}}$ \\
\hline $0.1 \%$ Clove bud oil $+3 \%$ Apple extract & $1.31 \pm 0.59^{\mathrm{FG}}$ & $2.33 \pm 1.02^{\mathrm{A}}$ & $1.95 \pm 1.07^{\mathrm{CDE}}$ & $1.45 \pm 0.77^{\mathrm{DEF}}$ & $1.52 \pm 0.79^{\mathrm{ABC}}$ \\
\hline $0.1 \%$ Lemongrass oil + $1 \%$ Apple extract & $2.03 \pm 1.14^{\mathrm{E}}$ & $1.60 \pm 0.74^{\mathrm{BCD}}$ & $1.92 \pm 1.02^{\mathrm{CDE}}$ & $2.00 \pm 1.15^{\mathrm{BCD}}$ & $1.98 \pm 1.21^{\mathrm{AB}}$ \\
\hline $0.1 \%$ Oregano oil + $1 \%$ Grapeseed extract & $2.45 \pm 1.08^{\mathrm{BCDE}}$ & $2.20 \pm 1.04^{\mathrm{A}}$ & $2.15 \pm 1.20^{\mathrm{BCD}}$ & $2.39 \pm 1.10^{\mathrm{ABC}}$ & $1.80 \pm 0.99^{\mathrm{ABC}}$ \\
\hline $0.1 \%$ Oregano oil $+7 \%$ olive extract & $3.18 \pm 1.19^{\mathrm{A}}$ & $1.98 \pm 0.85^{\mathrm{AB}}$ & $3.00 \pm 1.34^{\mathrm{A}}$ & $2.97 \pm 1.26^{\mathrm{A}}$ & $2.00 \pm 1.20^{\mathrm{AB}}$ \\
\hline
\end{tabular}

\subsection{Comparison of Spinach and Iceberg Lettuce with Regard to Sensory Analysis}

Overall, most of the treatments for spinach samples had a higher preference liking by consumers in comparison to those for lettuce samples. When panelists were asked which leafy green was preferred/consumed the most, spinach was also rated the highest and this could have influenced the preference liking for spinach samples after treatment. Additionally, plant antimicrobials had a lower impact on the color and texture properties of organic baby spinach in comparison to iceberg lettuce; therefore, this could be a factor contributing to the lower preference rating for iceberg lettuce samples. Combination treatments such as $0.1 \%$ clove bud oil $+3 \%$ apple extract had a rating of $3.5 \pm 1.99$ (dislike moderately) for iceberg lettuce; however, the same treatment had a rating of $5.7 \pm 2.4$ (neither like nor dislike) for baby spinach. A similar trend was seen with the combination treatment of $0.1 \%$ oregano oil $+1 \%$ grapeseed extract and the individual treatment of $7 \%$ olive extract, where the preference rating based on the overall acceptability was increased by 1-unit scale on spinach samples in comparison to iceberg lettuce.

It was evident that the combination treatments did not increase the preference liking among consumers for both iceberg lettuce and spinach samples. In general, a concentration-dependent effect on the preference liking was observed. For example, in case of iceberg lettuce samples, the treatment with $3 \%$ apple extract was rated $4.8 \pm 2.1$ (dislike slightly) and when $3 \%$ apple extract was combined with $0.1 \%$ clove bud oil, the overall acceptability dropped by about 1 unit (3.5 \pm 2.0 , dislike moderately). However, when lower concentrations of extracts were used in combination with essential oils, then the preference liking slightly increased. Treatment with $0.1 \%$ oregano oil in combination with $1 \%$ grapeseed extract had a preference liking of $4.0 \pm 2.3$ (dislike slightly), which was better than that of $0.1 \%$ clove bud oil $+3 \%$ apple extract; however, using plant extracts or essential oils alone in general had higher preference liking. Treatment of $0.1 \%$ clove bud oil alone for iceberg lettuce showed a preference rating of $6.0 \pm 2.0$ (like slightly) and $3 \%$ apple extract had a rating of $4.8 \pm 2.1$ (dislike slightly).

Linear regression (Figure 2) was conducted to compare the data on the ranking of overall acceptability with aroma, color, freshness, mouthfeel, and flavor. For iceberg lettuce samples, flavor $\left(\mathrm{R}^{2}=0.91\right)$ and mouthfeel $\left(\mathrm{R}^{2}=0.92\right)$ parameters were closely related to the rating of overall acceptability (Figure 2(a)) for all treatments. 


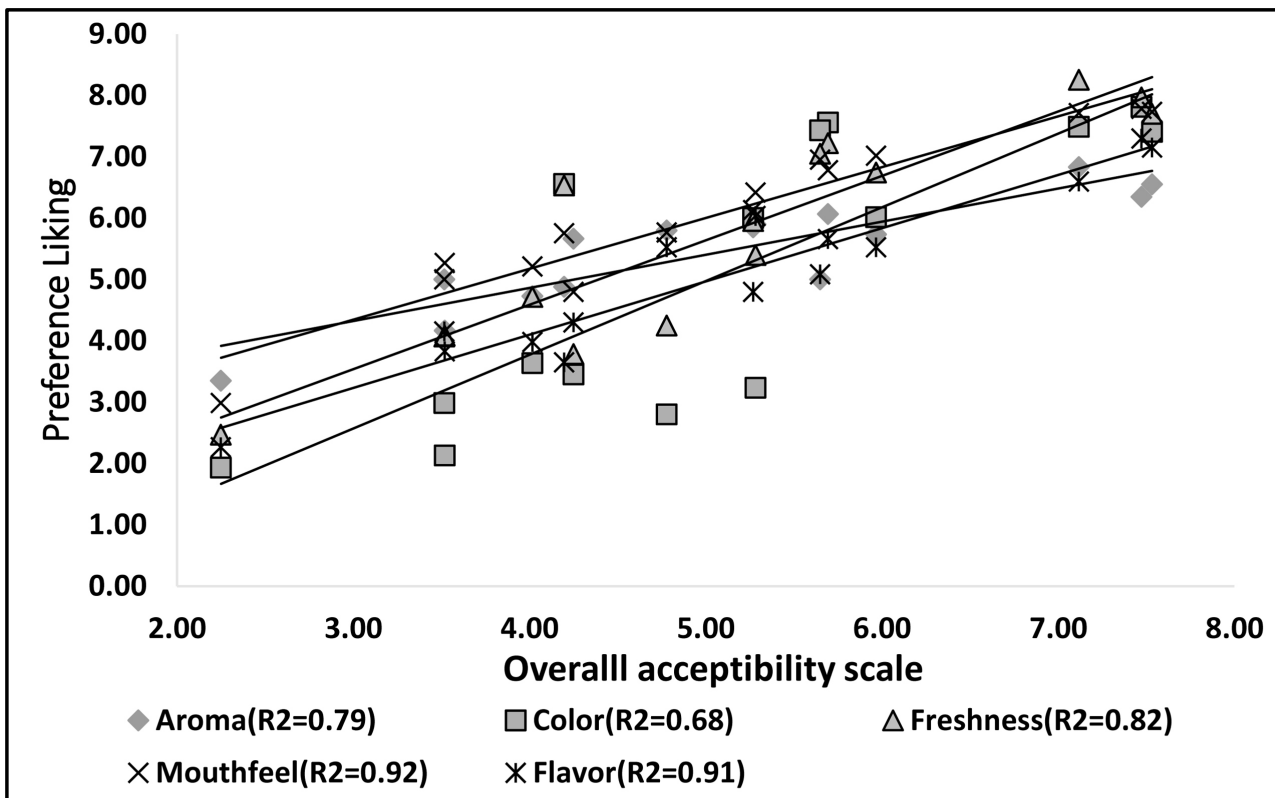

(a)

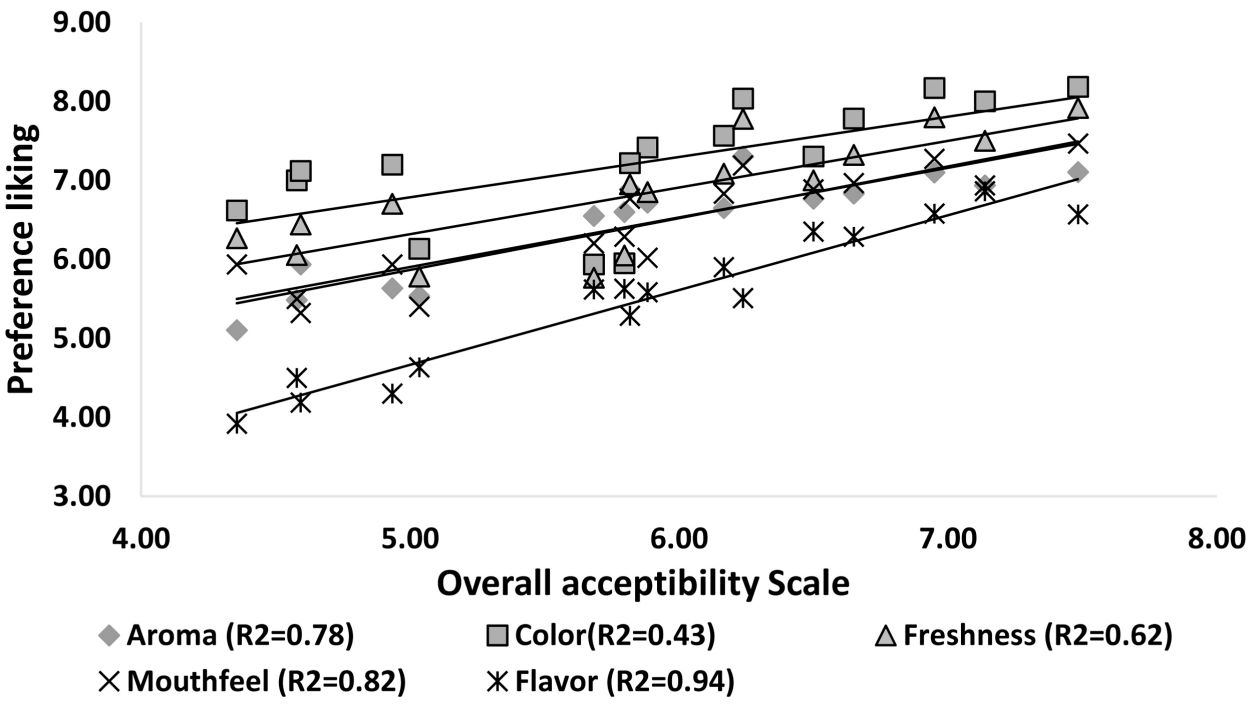

(b)

Figure 2. Linear correlation between values of average overall acceptability rating and other sensory parameters (aroma, freshness, mouthfeel, color, and flavor) after treatment with plant antimicrobials for iceberg lettuce (a) and baby spinach (b) samples.

For spinach samples, flavor $\left(\mathrm{R}^{2}=0.94\right)$ was the most influencing factor in determining the overall acceptability of a sample suggested by the strong correlation value (Figure 2(b)). A similar trend was seen where a correlation of $\mathrm{R}^{2}=0.91$ was found between flavor and overall acceptability of a salad dressing incorporated with $0.2 \%$ oregano EO and $1.14 \%$ salt [16]. In some cases, a strong correlation was seen between color attributes and overall acceptability for lettuce samples washed with plant extracts. For example, iceberg lettuce leaf washed with a combination of $0.1 \%$ oregano oil $+10 \%$ olive extract had the highest correlation of $\mathrm{R}^{2}=0.80$ between color and overall acceptability (data not shown). In general, for all treatments, upon comparing overall acceptability with other sensory parameters (aroma, color, freshness, mouthfeel, and flavor), flavor was the most influencing factor determining the overall acceptability of samples as indicated by Figure 2(a) and Figure 2(b). 
Additionally, each panelist was asked about the likelihood of purchasing leafy greens washed with a specific treatment. In response to this question, iceberg lettuce or spinach treated with $0.1 \%$ cinnamon oil was very/extremely likely $(25-26 / 60)$ to be purchased by panelists (data not shown). A strong correlation was seen between the rating of overall acceptability and likelihood of purchasing for both spinach $\left(\mathrm{R}^{2}=0.95\right)$ and iceberg lettuce $\left(\mathrm{R}^{2}=0.96\right)$ after washing with plant antimicrobials (data not shown).

\subsection{Effects of Plant Antimicrobial Treatments on the Texture of Organic Leafy Greens}

Textural changes are one of the main causes of quality loss in a food product [17]. It is therefore crucial to evaluate the impact of plant antimicrobial treatments on organic leafy greens. It is difficult however, to obtain texture measurements on iceberg lettuce because of the heterogeneity of lettuce leaves. Iceberg lettuce has two types of tissues, vascular and photosynthetic, which are relatively different in textural properties [29]. In the present study, we have shown that overall, plant antimicrobials had a higher impact on the textural properties of iceberg lettuce in comparison to spinach, due to the softer tissues and non-uniformity in the texture of iceberg lettuce.

Texturometer analysis showed that iceberg lettuce washed with calcium lactate had significantly higher $(\mathrm{p}>$ 0.05 ) crispiness value than samples washed with chlorine [17]. All plant antimicrobial treatments for iceberg lettuce had higher firmness values (Table 5) than control, except the individual treatments of $0.1 \%$ lemongrass oil and 3\% apple extract. These results indicate that some plant antimicrobials are able to improve the textural properties of iceberg lettuce in comparison to the control (tap water wash). Our results showed that treatments with cinnamon oil, clove bud oil, citral, grapeseed extract, and carvacrol may enhance the textural properties of organic leafy greens because greater force was required to crush these samples, and also panelists rated these treatments higher for freshness quality.

Table 5. Crispiness/Peak Force values of organic iceberg lettuce and baby spinach samples after washing with plant-antimicrobials and storage at $4{ }^{\circ} \mathrm{C}$ for $24 \mathrm{~h}$. Crispiness values were measured by taking an average of the highest peak force $(\mathrm{N})$ required to crush the samples from three separate trials \pm standard deviation. Different letters signify statistical differences $(\mathrm{p} \leq$ 0.05 ) between force values of various treatments.

\begin{tabular}{|c|c|c|}
\hline Treatment & Peak Force Lettuce (N) & Peak Force Spinach (N) \\
\hline Control & $575.8 \pm 82.1^{\mathrm{C}}$ & $845.00 \pm 5.84^{\mathrm{ABCD}}$ \\
\hline $3 \% \mathrm{H}_{2} \mathrm{O}_{2}$ & $663.1 \pm 53.9^{\mathrm{ABC}}$ & $893 \pm 45.8^{\mathrm{ABC}}$ \\
\hline $0.1 \%$ Cinnamon oil & $607.9 \pm 26.5^{\mathrm{BC}}$ & $933.5 \pm 43.5^{\mathrm{AB}}$ \\
\hline $0.1 \%$ Clove bud oil & $663.6 \pm 36.0^{\mathrm{ABC}}$ & $898.1 \pm 65.9^{\mathrm{ABC}}$ \\
\hline $0.1 \%$ Lemongrass oil & $574.4 \pm 49.8^{\mathrm{C}}$ & $885.3 \pm 51.7^{\mathrm{ABCD}}$ \\
\hline $0.1 \%$ Oregano oil & $649.7 \pm 60.1^{\mathrm{ABC}}$ & $774.7 \pm 42.1^{\mathrm{D}}$ \\
\hline $0.1 \%$ Carvacrol & $665.1 \pm 23.5^{\mathrm{ABC}}$ & $822.8 \pm 22.1^{\mathrm{BCD}}$ \\
\hline $0.1 \%$ Citral & $631.1 \pm 19.1^{\mathrm{BC}}$ & $891.8 \pm 46.9^{\mathrm{ABC}}$ \\
\hline 3\% Apple extract & $571.6 \pm 60.0^{\mathrm{C}}$ & $924.9 \pm 35.6^{\mathrm{ABC}}$ \\
\hline 3\% Grapeseed extract & $721.0 \pm 29.2^{\mathrm{AB}}$ & $845 \pm 10.43^{\mathrm{ABCD}}$ \\
\hline $7 \%$ Olive extract & $630.3 \pm 51.9^{\mathrm{BC}}$ & $893.4 \pm 22.9^{\mathrm{ABC}}$ \\
\hline $10 \%$ Olive Extract & $718.5 \pm 36.7^{\mathrm{AB}}$ & ND \\
\hline $0.1 \%$ Cinnamon oil $+1 \%$ Grapeseed extract & $\mathrm{ND}^{*}$ & $904.23 \pm 3.09^{\mathrm{ABC}}$ \\
\hline $0.1 \%$ Clove bud oil $+3 \%$ Apple extract & $667.6 \pm 50.3^{\mathrm{ABC}}$ & $854.8 \pm 59.4^{\mathrm{ABCD}}$ \\
\hline $0.1 \%$ Lemongrass oil + 1\% Apple extract & ND & $939.0 \pm 35.2^{\mathrm{A}}$ \\
\hline $0.1 \%$ Oregano oil + $1 \%$ Grapeseed extract & $657.7 \pm 22.9^{\mathrm{ABC}}$ & $818.0 \pm 18.0^{\mathrm{CD}}$ \\
\hline $0.1 \%$ Oregano oil $+7 \%$ Olive extract & ND & $844.9 \pm 19.2^{\mathrm{ABCD}}$ \\
\hline $0.1 \%$ Oregano oil $+10 \%$ Olive extract & $783.1 \pm 53.8^{\mathrm{A}}$ & ND \\
\hline
\end{tabular}

*ND-Measurements for these treatments were not done since these were not included in the sensory panels. 
For iceberg lettuce, treatment with $0.1 \%$ oregano oil $+10 \%$ olive extract combination yielded the highest force $(F=783.1 \pm 53.8 \mathrm{~N})$. This treatment significantly $(\mathrm{p} \geq 0.05)$ influenced the texture of iceberg lettuce by making the sample softer and increasing its elasticity. By visualization and tactile feel, this did not indicate crispiness; however, due to an increase in elasticity, this sample might have needed a higher force to be crushed in comparison to other treatments. The treatment of iceberg lettuce with $0.1 \%$ oregano oil in combination with $10 \%$ olive extract was also rated the lowest by consumers for mouthfeel and freshness (Table 1 ), indicating that this treatment significantly influenced the textural properties of iceberg lettuce. The firmness of spinach was not very much affected by plant antimicrobial treatments in comparison to iceberg lettuce, since spinach is thicker and more uniform in textural properties than iceberg lettuce. Additionally, the weight of iceberg lettuce is more water-based than spinach. The treatment with $0.1 \%$ oregano oil on spinach had the greatest loss in firmness $(\mathrm{F}=$ $774.7 \pm 42.1 \mathrm{~N}$ ) as indicated by the least force required to crush the samples in comparison to other treatments. Cinnamon oil may improve the textural properties of iceberg lettuce as shown by our results and it had higher crispiness value $(\mathrm{F}=607.9 \pm 26.5 \mathrm{~N})$ compared to the control $(\mathrm{F}=575.8 \pm 82.1 \mathrm{~N})$ (Table 5).

For spinach samples, in general, plant extracts had less impact on the firmness quality than EOs, even though no significant $(\mathrm{p} \leq 0.05)$ difference was observed. Additionally, combination treatments of plant extracts and EOs improved the firmness of spinach leaves, as greater force was required to crush these samples. Combination treatments such as $0.1 \%$ lemongrass oil $+1 \%$ apple extract $(\mathrm{F}=939 \pm 35.2 \mathrm{~N})$, and $0.1 \%$ cinnamon oil $+1 \%$ grapeseed extract $(\mathrm{F}=904.23 \pm 3.09 \mathrm{~N})$ enhanced the firmness quality of spinach samples. Plant extracts in combination with EOs may form a complex that perhaps may prevent EOs from coming directly into contact with the leaf, thus preventing any adverse effects on the organoleptic properties. One way to minimize the organoleptic effects of EOs is via the formation of nanoemulsions that could help improve EO stability and antimicrobial activity [30]. Studies have shown that a combination of essential oils with other treatments may act synergistically to improve their antimicrobial activity and thus may help reduce the concentrations of EOs to prevent any adverse impact on the sensory properties of the food product [13].

\subsection{Impact of Plant Antimicrobial Treatments on the Color of Organic Leafy Greens}

The color properties of spinach samples were not significantly $(\mathrm{p} \leq 0.05)$ affected by plant antimicrobials because spinach is much darker in color compared to iceberg lettuce (Table 6 and Table 7). Our data have also indicated that spinach samples had more negative $a^{*}$ values in comparison to iceberg samples, indicating a dark

Table 6. CIE L*, a*, b* coordinates obtained using Chroma Meter for antimicrobial treated organic iceberg lettuce. Average values, which do not share the same letter, are significantly different $(\mathrm{p} \leq 0.05)$.

\begin{tabular}{|c|c|c|c|}
\hline Treatments & $\mathbf{L}^{*}$ & $a^{*}$ & $\mathbf{b}^{*}$ \\
\hline Control & $58.38 \pm 4.58^{\mathrm{A}}$ & $-6.50 \pm 3.47^{\mathrm{C}}$ & $14.94 \pm 7.13^{\mathrm{A}}$ \\
\hline $\mathrm{H}_{2} \mathrm{O}_{2}$ & $59.03 \pm 5.05^{\mathrm{A}}$ & $-8.15 \pm 4.88^{\mathrm{C}}$ & $18.71 \pm 7.83^{\mathrm{A}}$ \\
\hline $0.1 \%$ Cinnamon oil & $57.06 \pm 5.15^{\mathrm{AB}}$ & $-4.22 \pm 3.04^{\mathrm{ABC}}$ & $9.69 \pm 7.31^{\mathrm{A}}$ \\
\hline 0.1\% Clove bud oil & $58.20 \pm 3.95^{\mathrm{A}}$ & $-5.02 \pm 4.07^{\mathrm{ABC}}$ & $11.75 \pm 9.04^{\mathrm{A}}$ \\
\hline $0.1 \%$ Lemongrass oil & $48.39 \pm 9.37^{\mathrm{BC}}$ & $-5.42 \pm 3.96^{\mathrm{BC}}$ & $15.13 \pm 7.37^{\mathrm{A}}$ \\
\hline $0.1 \%$ Oregano oil & $51.75 \pm 8.38^{\mathrm{ABC}}$ & $-5.10 \pm 2.87^{\mathrm{ABC}}$ & $14.28 \pm 6.47^{\mathrm{A}}$ \\
\hline $0.1 \%$ Carvacrol & $51.32 \pm 5.69^{\mathrm{ABC}}$ & $-4.35 \pm 5.80^{\mathrm{ABC}}$ & $16.08 \pm 6.62^{\mathrm{A}}$ \\
\hline $0.1 \%$ Citral & $54.01 \pm 9.21^{\mathrm{AB}}$ & $-6.19 \pm 2.94^{\mathrm{BC}}$ & $14.79 \pm 5.97^{\mathrm{A}}$ \\
\hline 3\% Apple extract & $52.84 \pm 5.17^{\mathrm{ABC}}$ & $-3.35 \pm 4.26^{\mathrm{ABC}}$ & $16.29 \pm 8.34^{\mathrm{A}}$ \\
\hline 3\% Grapeseed extract & $55.63 \pm 5.36^{\mathrm{AB}}$ & $0.10 \pm 2.97^{\mathrm{A}}$ & $18.74 \pm 6.21^{\mathrm{A}}$ \\
\hline $7 \%$ Olive extract & $54.40 \pm 5.66^{\mathrm{AB}}$ & $-5.44 \pm 3.97^{\mathrm{BC}}$ & $19.20 \pm 8.29^{\mathrm{A}}$ \\
\hline $10 \%$ Olive extract & $55.05 \pm 7.39^{\mathrm{AB}}$ & $-2.47 \pm 2.10^{\mathrm{ABC}}$ & $17.41 \pm 5.76^{\mathrm{A}}$ \\
\hline $0.1 \%$ Clove bud oil $+3 \%$ Apple extract & $56.31 \pm 3.56^{\mathrm{AB}}$ & $-4.86 \pm 4.78^{\mathrm{ABC}}$ & $19.38 \pm 6.17^{\mathrm{A}}$ \\
\hline $0.1 \%$ Oregano oil $+1 \%$ Grapeseed extract & $49.20 \pm 6.31^{\mathrm{BC}}$ & $-2.92 \pm 2.89^{\mathrm{ABC}}$ & $13.67 \pm 5.34^{\mathrm{A}}$ \\
\hline $0.1 \%$ Oregano oil $+10 \%$ Olive extract & $44.49 \pm 6.21^{\mathrm{C}}$ & $-0.93 \pm 3.32^{\mathrm{AB}}$ & $16.94 \pm 4.38^{\mathrm{A}}$ \\
\hline
\end{tabular}


Table 7. CIE L ${ }^{*}, a^{*}, b^{*}$ coordinates obtained using Chroma Meter for antimicrobial treated baby spinach. Average values which do not share the same letter, are significantly different $(\mathrm{p} \leq 0.05)$.

\begin{tabular}{|c|c|c|c|}
\hline Treatments & $\mathbf{L}^{*}$ & $a^{*}$ & $\mathbf{b}^{*}$ \\
\hline Control & $37.48 \pm 2.90^{\mathrm{ABC}}$ & $-13.84 \pm 1.73^{\mathrm{AB}}$ & $18.63 \pm 3.38^{\mathrm{A}}$ \\
\hline $3 \% \mathrm{H}_{2} \mathrm{O}_{2}$ & $38.54 \pm 1.65^{\mathrm{AB}}$ & $-14.26 \pm 1.97^{\mathrm{AB}}$ & $19.84 \pm 3.84^{\mathrm{A}}$ \\
\hline $0.1 \%$ Cinnamon oil & $39.26 \pm 1.63^{\mathrm{AB}}$ & $-13.61 \pm 1.52^{\mathrm{AB}}$ & $19.37 \pm 1.85^{\mathrm{A}}$ \\
\hline $0.1 \%$ Clove bud oil & $35.87 \pm 4.42^{\mathrm{ABC}}$ & $-13.92 \pm 1.51^{\mathrm{AB}}$ & $18.91 \pm 2.92^{\mathrm{A}}$ \\
\hline $0.1 \%$ Lemongrass oil & $34.63 \pm 4.95^{\mathrm{BC}}$ & $-14.49 \pm 2.13^{\mathrm{B}}$ & $18.40 \pm 2.57^{\mathrm{A}}$ \\
\hline $0.1 \%$ Oregano oil & $32.87 \pm 3.69^{\mathrm{C}}$ & $-14.36 \pm 1.51^{\mathrm{B}}$ & $18.63 \pm 2.34^{\mathrm{A}}$ \\
\hline $0.1 \%$ Carvacrol & $34.73 \pm 3.81^{\mathrm{BC}}$ & $-14.29 \pm 2.28^{\mathrm{B}}$ & $18.18 \pm 2.58^{\mathrm{A}}$ \\
\hline $0.1 \%$ Citral & $38.02 \pm 3.49^{\mathrm{AB}}$ & $-14.13 \pm 1.78^{\mathrm{B}}$ & $18.93 \pm 4.01^{\mathrm{A}}$ \\
\hline 3\% Apple extract & $38.25 \pm 2.64^{\mathrm{AB}}$ & $-12.88 \pm 1.34^{\mathrm{AB}}$ & $17.61 \pm 2.70^{\mathrm{A}}$ \\
\hline 3\% Grapeseed extract & $37.47 \pm 5.02^{\mathrm{ABC}}$ & $-12.16 \pm 2.10^{\mathrm{AB}}$ & $20.97 \pm 4.74^{\mathrm{A}}$ \\
\hline $7 \%$ Olive extract & $40.27 \pm 4.32^{\mathrm{A}}$ & $-13.68 \pm 1.71^{\mathrm{AB}}$ & $21.28 \pm 4.79^{\mathrm{A}}$ \\
\hline $\begin{array}{c}\text { 0.1\% Cinnamon oil }+1 \% \\
\text { Grapeseed extract }\end{array}$ & $37.45 \pm 3.19^{\mathrm{ABC}}$ & $-10.60 \pm 6.83^{\mathrm{A}}$ & $17.77 \pm 4.25^{\mathrm{A}}$ \\
\hline $\begin{array}{c}0.1 \% \text { Clove bud oil }+3 \% \\
\text { Apple extract }\end{array}$ & $38.71 \pm 1.42^{\mathrm{AB}}$ & $-12.56 \pm 1.16^{\mathrm{AB}}$ & $18.22 \pm 2.88^{\mathrm{A}}$ \\
\hline $\begin{array}{c}0.1 \% \text { Lemongrass oil }+1 \% \\
\text { Apple extract }\end{array}$ & $38.27 \pm 3.65^{\mathrm{AB}}$ & $-13.60 \pm 1.20^{\mathrm{AB}}$ & $18.90 \pm 3.88^{\mathrm{A}}$ \\
\hline $\begin{array}{l}0.1 \% \text { Oregano oil }+1 \% \\
\text { Grapeseed extract }\end{array}$ & $37.17 \pm 2.33^{\mathrm{ABC}}$ & $-12.42 \pm 2.16^{\mathrm{AB}}$ & $20.22 \pm 2.39^{\mathrm{A}}$ \\
\hline $\begin{array}{l}0.1 \% \text { Oregano oil }+7 \% \\
\text { Olive extract }\end{array}$ & $37.34 \pm 2.46^{\mathrm{ABC}}$ & $-13.70 \pm 1.81^{\mathrm{AB}}$ & $18.87 \pm 3.53^{\mathrm{A}}$ \\
\hline
\end{tabular}

green color for spinach (Table 7). When panelists evaluated these leafy greens, the preference liking rankings were much higher for spinach samples than iceberg lettuce for color attributes. For iceberg samples, the treatment that showed the greatest impact on color properties was a combination of $0.1 \%$ oregano oil with $10 \%$ olive extract. This treatment was the least preferred by panelists on the basis of color and rated the highest for browning on iceberg lettuce. The combination of $0.1 \%$ oregano oil with $10 \%$ olive extract had the lowest $\mathrm{L}^{*}$ value of $44.5 \pm 6.2$ (Table 6) among all treatments, indicating that this sample had the darkest color among lettuce samples. Additionally, the treatment with $3 \%$ grapeseed extract on iceberg lettuce had $a^{*}=0.10 \pm 3.0$ indicating a slight reddish color that was also seen visually by panelists. Higher impact on color change may be evident in combination treatments rather than on individual treatments. A higher impact on color was found when iceberg lettuce was washed in ozonated water containing calcium lactate in comparison to individual treatments [31]. In the present study, all other plant antimicrobial treatments did not have a significant $(p \leq 0.05)$ impact on the color properties of iceberg lettuce or spinach.

\section{Conclusion}

This study provides useful information about plant antimicrobials that could potentially be used as organic sanitizers, while having a low impact on the organoleptic properties of organic leafy greens. Organic leafy greens treated with $0.1 \%$ cinnamon oil had the highest preference liking by panelists. This treatment also had the least impact on the sensory properties of both spinach and lettuce; therefore, cinnamon oil may be a good alternative to chemical sanitizers. In general, treatments with EOs were rated high for pungency and off-odor, whereas treatments with plant extracts were rated high for browning. Combination treatments of plant extracts with essential oils were the least preferred, so alternative treatments need to be evaluated. Our results have shown that certain types of leafy greens such as baby spinach may have higher preference liking after washing with plant antimicrobials; however, additional research needs to be conducted. Future studies will focus on conducting 
sensory analysis of organic leafy greens with trained panelists to reduce the variability in sensory data. The influence of plant antimicrobials on the sensory properties of other organic leafy greens such as romaine lettuce merits further investigation. In the end, practical application of effective plant antimicrobials will be highly influenced by their sensory effects on foods.

\section{Acknowledgements}

This work was supported by the U.S. Department of Agriculture, National Institute of Food and Agriculture, Organic Research and Extension Initiative competitive grant no. 2010-51300-21760. The authors would like to thank The Food Technology Corporation for providing the Kramer shear probe for texture analysis and Mr. Drew Lambert for his technical assistance with the texture analysis. The authors also thank Libin Zhu of the Ravishankar laboratory for his technical assistance and training the graduate student on data analysis. Additionally, we thank Rena Shifren, a sensory evaluation and consumer behavior specialist from Prosense Consumer and Research Center, Tucson, AZ, for her technical assistance with the sensory study; and Dr. Roberto de Jesús Avena Bustillos, USDA-ARS-WRRC-Albany, CA for his critical review of this manuscript.

\section{Conflict of Interest}

The authors of this manuscript have no conflict of interest to declare.

\section{References}

[1] Goldman, B.J. and Clancy, K.L. (1991) A Survey of Organic Produce Purchases and Related Attitudes of Food Cooperative Shoppers. American Journal of Alternative Agriculture, 6, 89-96. http://dx.doi.org/10.1017/S0889189300003933

[2] Makatouni, A. (2002) What Motivates Consumers to Buy Organic Food in the UK? Results from a Qualitative Study. British Food Journal, 104, 345-352. http://dx.doi.org/10.1108/00070700210425769

[3] Magnusson, M.K., Arvola, A., Hursti, U.K.K., Aberg, L. and Sjoden, P.O. (2003) Choice of Organic Foods Is Related to Perceived Consequences for Human Health and to Environmentally Friendly Behaviour. Appetite, 40, 109-117. http://dx.doi.org/10.1016/S0195-6663(03)00002-3

[4] United States Department of Agriculture Economic Research Service (USDA-ERS) (2016) Organic Market Overview. http://www.ers.usda.gov/topics/natural-resources-environment/organic-agriculture/organic-market-overview.aspx

[5] Oliveira, M., Usall, J., Vinas, I., Anguera, M., Gatius, F. and Abadias, M. (2010) Microbiological Quality of Fresh Lettuce from Organic and Conventional Production. Food Microbiology, 27, 679-684. http://dx.doi.org/10.1016/j.fm.2010.03.008

[6] Food and Drug Administration (2005) Food and Drugs (GRAS). Accessed 21 November 2005. http://www.fda.gov

[7] Burt, S. (2004) Essential Oils: Their Antibacterial Properties and Potential Applications in Foods: A Review. International Journal of Food Microbiology, 94, 223-253. http://dx.doi.org/10.1016/j.ijfoodmicro.2004.03.022

[8] Hsieh, P.-C., Mau, J.-L. and Huang, S.-H. (2001) Antimicrobial Effect of Various Combinations of Plant Extracts. Food Microbiology, 18, 35-43. http://dx.doi.org/10.1006/fmic.2000.0376

[9] Steptoe, A., Pollard, T.M. and Wardle, J. (1995) Development of a Measure of the Motives Underlying the Selection of Food: The Food Choice Questionnaire. Appetite, 25, 267-284. http://dx.doi.org/10.1006/appe.1995.0061

[10] Karabagias, I., Badeka, A. and Kontominas, M.G. (2011) Shelf Life Extension of Lamb Meat Using Thyme or Oregano Essential Oils and Modified Atmosphere Packaging. Meat Science, 88, 109-116. http://dx.doi.org/10.1016/j.meatsci.2010.12.010

[11] Roller, S. and Seedhar, P. (2002) Carvacrol and Cinnamic Acid Inhibit Microbial Growth in Fresh-Cut Melon and Kiwifruit at $4^{\circ} \mathrm{C}$ and $8^{\circ} \mathrm{C}$. Letters in Applied Microbiology, 35, 390-394. http://dx.doi.org/10.1046/j.1472-765X.2002.01209.x

[12] Chida, M., Yamashita, K., Izumiya, Y., Watanabe, K. and Tamura, H. (2006) Aroma Impact Compounds in Three Citrus Oils: Cross-Matching Test and Correspondence Analysis Approach. Journal of Food Science, 71, S54-S58. http://dx.doi.org/10.1111/j.1365-2621.2006.tb12406.x

[13] Gutierrez, J., Bourke, P., Lonchamp, J. and Barry-Ryan, C. (2009) Impact of Plant Essential Oils on Microbiological, Organoleptic and Quality Markers of Minimally Processed Vegetables. Innovative Food Science and Emerging Technologies, 10, 195-202. http://dx.doi.org/10.1016/j.ifset.2008.10.005 
[14] Espina, L., García-Gonzalo, D. and Pagán, R. (2014) Impact of Essential Oils on the Taste Acceptance of Tomato Juice, Vegetable Soup, or Poultry Burgers. Journal of Food Science, 79, S1575-S1583. http://dx.doi.org/10.1111/1750-3841.12529

[15] Barrett, D.M., Beaulieu, J.C. and Shewfelt, R. (2010) Color, Flavor, Texture, and Nutritional Quality of Fresh-Cut Fruits and Vegetables: Desirable Levels, Instrumental and Sensory Measurement, and the Effects of Processing. Critical Reviews in Food Science and Nutrition, 50, 369-389. http://dx.doi.org/10.1080/10408391003626322

[16] Cattelan, M., Castilhos, M., Silva, D. and Hoffmann, A. (2015) Oregano Essential Oil: Effect on Sensory Acceptability. Nutrition \& Food Science, 45, 574-582. http://dx.doi.org/10.1108/NFS-02-2015-0014

[17] Martin-Diana, A.B., Rico, D., Friaas, J., Henehan, G.T.M., Mulcahy, J., Barat, J.M. and Barry-Ryan, C. (2006) Effect of Calcium Lactate and Heat-Shock on Texture in Fresh-Cut Lettuce during Storage. Journal of Food Engineering, 77, 1069-1077. http://dx.doi.org/10.1016/j.jfoodeng.2005.08.037

[18] Du, W.X., Olsen, C.W., Avena-Bustillos, R.J., Friedman, M. and McHugh, T.H. (2011) Physical and Antibacterial Properties of Edible Films Formulated with Apple Skin Polyphenols. Journal of Food Science, 76, 149-155. http://dx.doi.org/10.1111/j.1750-3841.2010.02012.x

[19] Moore-Neibel, K., Gerber, C., Patel, J., Friedman, M., Jaroni, D. and Ravishankar, S. (2013) Antimicrobial Activity of Oregano Oil against Antibiotic-Resistant Salmonella enterica on Organic Leafy Greens at Varying Exposure Times and Storage Temperatures. Food Microbiology, 34, 123-129. http://dx.doi.org/10.1016/j.fm.2012.12.001

[20] Todd, J., Friedman, M., Patel, J., Jaroni, D. and Ravishankar, S. (2013) The Antimicrobial Effects of Cinnamon Leaf Oil against Multi-Drug Resistant Salmonella Newport on Organic Leafy Greens. International Journal of Food Microbiology, 166, 193-199. http://dx.doi.org/10.1016/j.ijfoodmicro.2013.06.021

[21] Ravishankar, S., Zhu, L., Reyna-Granados, J., Law, B., Joens, L. and Friedman, M. (2010) Carvacrol and Cinnamaldehyde Inactivate Antibiotic-Resistant Salmonella enterica in Buffer and on Celery and Oysters. Journal of Food Protection, 73, 234-240.

[22] Ravishankar, S., Zhu, L., Law, B., Joens, L. and Friedman, M. (2008) Plant-Derived Compounds Inactivate Antibiotic-Resistant Campylobacter jejuni Strains. Journal of Food Protection, 71, 1145-1149.

[23] Asensio, C.M., Nepote, V. and Grosso, N.R. (2012) Sensory Attribute Preservation in Extra Virgin Olive Oil with Addition of Oregano Essential Oil as Natural Antioxidant. Journal of Food Science, 77, 294-301. http://dx.doi.org/10.1111/j.1750-3841.2012.02841.x

[24] Lawless, H. and Heymann, H. (2010) Sensory Evaluation of Food Science Principles and Practices. Chapter 1, 2nd Edition, Ithaca, New York. http://dx.doi.org/10.1007/978-1-4419-6488-5

[25] Zhao, X., Chambers, I.V.E., Matta, Z., Loughin, T.M. and Carey, E.E. (2007) Consumer Sensory Analysis of Organically and Conventionally Grown Vegetables. Journal of Food Science, 72, 87-91. http://dx.doi.org/10.1111/j.1750-3841.2007.00277.x

[26] Drewnowski, A., Mennella, J.A., Johnson, S.L. and Bellisle, F. (2012) Sweetness and Food Preference. Journal of Nutrition, 142, 1142-1148. http://dx.doi.org/10.3945/jn.111.149575

[27] Wansink, B., Cheney, M.M. and Chan, N. (2003) Exploring Comfort Food Preferences across Age and Gender. Physiology and Behavior, 79, 739-747. http://dx.doi.org/10.1016/S0031-9384(03)00203-8

[28] De Azeredo, G.A., Stamford, T.L.M., Nunes, P.C., Gomes Neto, N.J., De Oliveira, M.E.G. and De Souza, E.L. (2011) Combined Application of Essential Oils from Origanum vulgare L. and Rosmarinus officinalis L. to Inhibit Bacteria and Autochthonous Microflora Associated with Minimally Processed Vegetables. Food Research International, 44, 1541-1548. http://dx.doi.org/10.1016/j.foodres.2011.04.012

[29] Toole, G.A., Parker, M.L., Smith, A.C. and Waldron, K.W. (2000) Mechanical Properties of Lettuce. Journal of Materials Science, 35, 3553-3559. http://dx.doi.org/10.1023/A:1004809428967

[30] Donsí, F., Annunziata, M., Sessa, M. and Ferrari, G. (2011) Nanoencapsulation of Essential Oils to Enhance Their Antimicrobial Activity in Foods. LWT-Food Science and Technology, 44, 1908-1914. http://dx.doi.org/10.1016/j.lwt.2011.03.003

[31] Rico, D., Martin-Diana, A.B., Henehan, G., Frias, J.M. and Barry-Ryan, C. (2006) Effect of Ozone and Calcium Lactate Treaments on Browning and Textured Properties of Fresh-Cut Lettuce. Journal of the Science of Food and Agriculture, 86, 2179-2188. http://dx.doi.org/10.1002/jsfa.2594 


\section{Submit or recommend next manuscript to SCIRP and we will provide best service for you:}

Accepting pre-submission inquiries through Email, Facebook, LinkedIn, Twitter, etc.

A wide selection of journals (inclusive of 9 subjects, more than 200 journals)

Providing 24-hour high-quality service

User-friendly online submission system

Fair and swift peer-review system

Efficient typesetting and proofreading procedure

Display of the result of downloads and visits, as well as the number of cited articles

Maximum dissemination of your research work

Submit your manuscript at: http://papersubmission.scirp.org/ 